\title{
Transverse Spin Asymmetries in the CNI Region of Elastic Proton-Proton Scattering at $\mathrm{s}=200 \mathrm{GeV}$
}

\author{
Dmitry Svirida \\ for the STAR Collaboration \\ Laboratory of Polarized Research, Institute for Theoretical and Experimental Physics, \\ 25 Bolshaya Cheremushkinskaya, Moscow, 117218, Russia \\ Dmitry.Svirida@itep.ru
}

Published 29 February 2016

\begin{abstract}
Precise measurements of transverse spin asymmetries in proton-proton elastic scattering at very small values of four-momentum transfer squared, $t$, have been performed using the Relativistic Heavy Ion Collider (RHIC) polarized proton beams. The measurements of both single and double spin asymmetries were made at the center-of-mass energy $\sqrt{s}=200 \mathrm{GeV}$ and in the region $0.003 \leq|t| \leq 0.035(\mathrm{GeV} / c)^{2}$, which was accessed using Roman Pot devices incorporated into the STAR experimental setup. The obtained set of asymmetries is sensitive to the poorly known hadronic contribution to the spinflip amplitudes and provide significant constraints for the theoretical descriptions of the reaction mechanism of proton-proton elastic scattering at high energies.
\end{abstract}

Keywords: Transverse spin asymmetry; elastic scattering; Coulomb-nuclear interference.

PACS numbers: 13.85.Dz, 13.88.+e

\section{Asymmetries and Helicity Amplitudes}

Measurements of the transverse spin asymmetries $A_{N}, A_{N N}$ and $A_{S S}$ provide important information on the spin dependence of the hadronic interactions at high energies and low momentum transfer. For the elastic proton-proton scattering the asymmetries are usually expressed in terms of helicity amplitudes, see Ref. 1 for details. With reasonable assumptions on the relative sizes of the amplitudes, approximate relations can be derived:

$A_{N} \frac{d \sigma}{d t} \approx-\frac{8 \pi}{s^{2}} \operatorname{Im} \phi_{5}^{*} \phi_{+}, \quad \frac{A_{N N}+A_{S S}}{2} \frac{d \sigma}{d t} \approx \frac{4 \pi}{s^{2}} \operatorname{Re} \phi_{1} \phi_{2}^{*}, \quad \frac{A_{N N}-A_{S S}}{2} \frac{d \sigma}{d t} \approx-\frac{4 \pi}{s^{2}} \operatorname{Re} \phi_{3} \phi_{4}^{*}$,

where $\phi_{+}=\left(\phi_{1}+\phi_{3}\right) / 2$ is the half-sum of dominating non-spin-flip amplitudes. For each amplitude, both electromagnetic and nuclear parts should be taken into

This is an Open Access article published by World Scientific Publishing Company. It is distributed under the terms of the Creative Commons Attribution 3.0 (CC-BY) License. Further distribution of this work is permitted, provided the original work is properly cited. 
account in the CNI region: $\phi_{i}=\phi_{i}^{h a d}+\phi_{i}^{e m}$. To quantify the spin-dependent hadronic contributions, the relative amplitudes are introduced:

$$
r_{2}=\frac{\phi_{2}^{h a d}}{2 \operatorname{Im} \phi_{+}^{h a d}}, \quad r_{4}=\frac{m_{p}^{2}}{-t} \frac{\phi_{4}^{h a d}}{\operatorname{Im} \phi_{+}^{h a d}} \quad \text { and } \quad r_{5}=\frac{2 m_{p}}{\sqrt{-t}} \frac{\phi_{5}^{h a d}}{\operatorname{Im} \phi_{+}^{h a d}} .
$$

The relative amplitudes include main kinematic factors and their dependence on momentum transfer is usually neglected in narrow $t$-ranges.

\section{Experimental Setup}

The experimental setup included two pairs of Roman Pot (RP) stations, located on both sides of the STAR detector at a distance of approximately $55 \mathrm{~m}$ from the interaction point (IP), see Fig. 1. Transversely polarized protons of colliding RHIC beams that scatter elastically at small angles at the IP follow the beam optics but got spatially separated from the beam at the location of the Roman Pots. The silicon strip detector planes inside the RPs approached the beam as close as $10 \mathrm{~mm}$ both from vertical and horizontal directions and provide full $2 \pi$ acceptance in the azimuthal angle for a $(-t)$-range of $0.003-0.035(\mathrm{GeV} / \mathrm{c})^{2}$. Further details of the setup can be found in Ref. 2. The Roman Pot detectors were incorporated into the STAR infrastructure thus giving full advantage of using other STAR subsystems for crosschecks and precise luminosity monitoring.

\section{Single Spin Asymmetry $\boldsymbol{A}_{N}$}

An extremely clean data sample of $2 \cdot 10^{7}$ elastic events was obtained during four fills of RHIC running with dedicated beam optics. Transverse polarization of both RHIC beams was as high as $60 \%$. The details of the elastic event selection procedure can be found in Ref. 2. The data was divided into five intervals in momentum transferred, while the background levels varied in the range $0.2-3 \%$ dependent on the $t$-interval.

For the extraction of the single spin asymmetry $A_{N}$ so-called 'square root formula' ${ }^{3}$ was used. This approach allows to avoid direct luminosity measurements since relative luminosities fully cancel out together with the detector asymmetries.

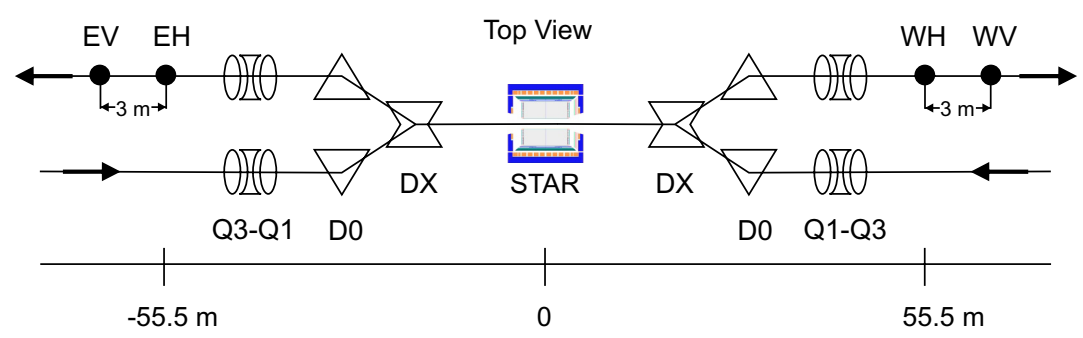

Fig. 1. Horizontal (EH, WH) and vertical (EV, WV) roman pot stations on both outgoing beams of the STAR interaction point. 

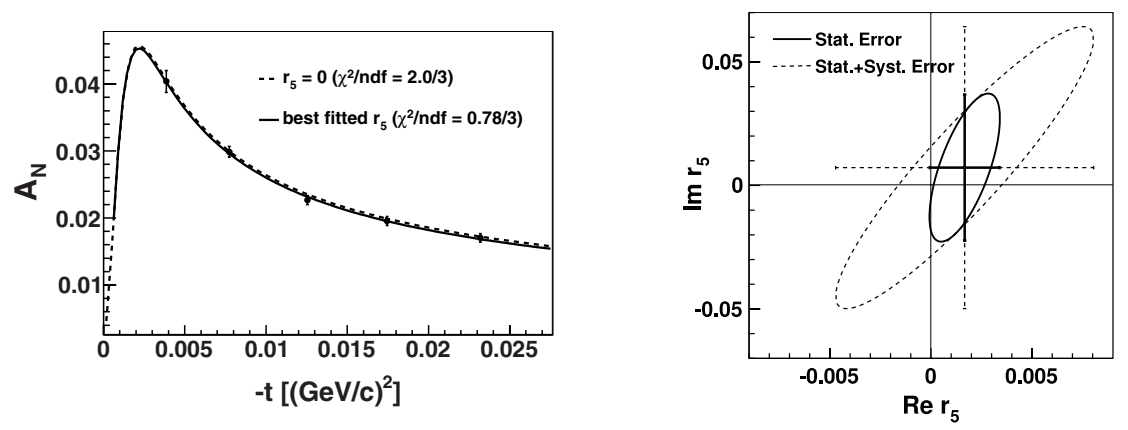

Fig. 2. Left - single spin asymmetry $A_{N}$ (points with errors), prediction with $r_{5}=0$ (dashed line), best $r_{5}$ fit (solid line); right - extracted values of $r_{5}$ with statistical and total uncertainties.

The measured values of $A_{N}$ are shown in the left panel of Fig. 2 together with parametrization based on relative amplitude $r_{5}$ : the dashed line corresponds to no hadronic single spin-flip contribution, i.e. $r_{5}=0$, while the solid line is the result of the fit using $r_{5}$ as a free parameter.

The right panel of Fig. 2 presents the complex value of $r_{5}$ with the $1 \sigma$ confidence ellipses for purely statistical error (solid line) and total uncertainty (dashed line). The latter was obtained through the accurate error matrix approach and includes a number of systematic factors, of which the beam polarization uncertainty is of most influence. The extracted value of $r_{5}$ is compatible with zero. Thus all of the experimental results, including those previously obtained in the range $6.8 \mathrm{Gev} \leq$ $\sqrt{s} \leq 200 \mathrm{GeV}$, are consistent with the assumption of no hadronic single spin-flip contribution to the elastic proton-proton scattering.

\section{Relative Luminosity}

For the double spin effects the azimuthal angular distribution of the raw asymmetry can be expressed in the form:

$$
A_{2}(\varphi)=P_{B} P_{Y}\left(\left(A_{N N}+A_{S S}\right) / 2+\left(A_{N N}-A_{S S}\right) / 2 \cdot \cos 2 \varphi\right)
$$

Contrary to the single spin case, the constant term in this expression does not allow to take advantage of the 'square root formula'. Instead, the only way to perform the analysis is to use event counts, normalized by an external luminosity monitor.

In order to obtain unbiased values of asymmetries the luminosity monitor must be free of spin-dependent effects. Several STAR subsystems were investigated for monitoring purposes and beam-beam counters (BBCs) were selected for the most precise luminosity measurements. $\mathrm{BBCs}^{4}$ are arrays of scintillator tiles surrounding the beam pipe on both sides of the STAR detector magnet, see Fig. 3, left panel. Three subprocesses inside BBC with substantially different physics were compared to each other and to the $\mathrm{BBC}$ as a whole in terms of the sensitivity to spin effects:

- exactly one hit in the 'inner' tiles, i.e. single particle at small scattering angle; 

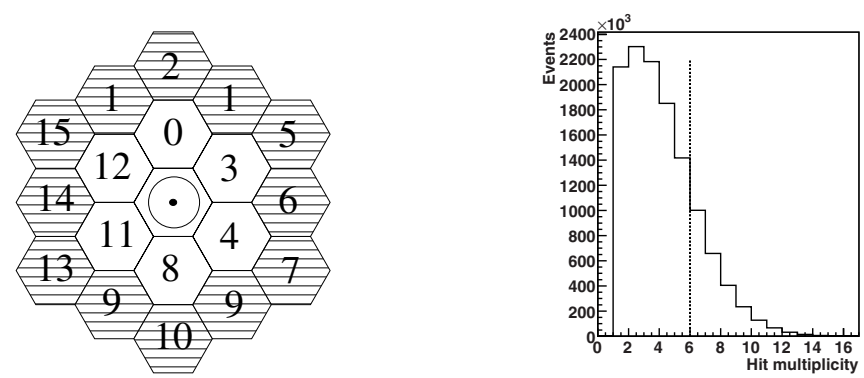

Fig. 3. Left - STAR BBC small tiles: white inner, hatched outer, the central circle and the dot show the beam pipe and the beam; right - hit multiplicity distribution in STAR BBC .

- exactly one hit in the 'outer' tiles, i.e. single particle at larger angle, on average twice as large as for the previous case;

- more than 5 hits in one arm, i.e. large forward multiplicity, see Fig. 3, right panel.

The resulting uncertainty on the double spin asymmetry, estimated from the subprocess comparison was found to be $8.4 \cdot 10^{-4}$.

\section{Background Subtraction}

Many stability tests were performed to proof the highest accuracy of the double spin asymmetry result. The only test, which showed significant sensitivity, was that by varying the $\chi_{\text {cut }}^{2}$ value, the main criterion for the elastic event selection. This led to detailed investigations of the background issues.

The experimental $\chi^{2}$ distribution (magnified background part) of the elastic angular correlation hypothesis is presented on the left of Fig. 4. See Ref. 2 for details about $\chi^{2}$ criterion. The common assumption about backgrounds in the polarization experiments is that the measured value of an asymmetry contains a component originating from the background process, which is proportional to the fraction of background events and the background asymmetry. Events with $20<\chi^{2}<60$
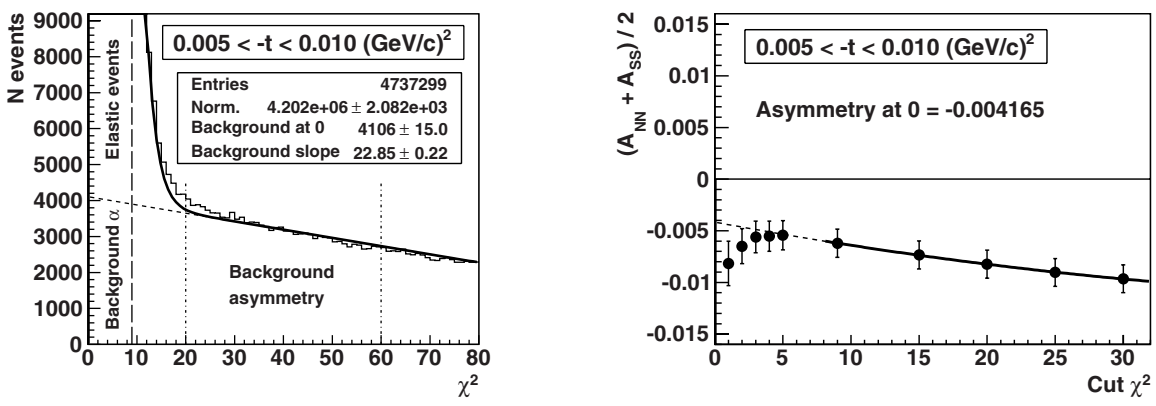

Fig. 4. Left - Event $\chi^{2}$ distribution for one of the $t$ intervals with areas of elastic event selection and background definition; right - extrapolation of $\left(A_{N N}+A_{S S}\right) / 2$ values to the limit of zero background. 
were chosen for background asymmetry calculations (dash-dotted lines in Fig. 4 (left)), while events were considered elastic if $\chi^{2}<\chi_{\text {cut }}^{2}=9$ (dashed line) and the background was extrapolated to this region to estimate its fractional amount. This common approach allows to accurately trace the statistical error, but stays disputable in terms of its systematic uncertainties because of unpredictable background properties (low fraction, but high fake asymmetries).

Another method developed in this analysis relies on the fact that the contribution of the background diminishes with decreasing value of $\chi_{c u t}^{2}$, and as $\chi_{\text {cut }}^{2} \rightarrow 0$ it reaches the zero limit. The evolution of the asymmetry results as a function of $\chi_{c u t}^{2}$ is illustrated in Fig. 4 (right). Extrapolation of the obtained behavior to $\chi_{\text {cut }}^{2}=0$ gives the best estimate of the asymmetries with no background contribution. The difference of the asymmetry values from the two methods was used as a measure of systematic uncertainty in individual $t$-intervals of the experiment.

\section{Double Spin Asymmetries}

The asymmetries obtained for the five $t$-intervals are plotted in Fig. 5. Boxes represent pure statistical errors, while the bars include the uncertainties from the background subtraction. All points of $\left(A_{N N}-A_{S S}\right) / 2$ (right) do not deviate from zero more than by two standard errors and the deviations are of different signs. The average is well compatible with zero. On the contrary, $\left(A_{N N}+A_{S S}\right) / 2$ (left) is significantly below zero and the absolute values are of the order of $5 \cdot 10^{-3}$. The distribution is approximately flat and the average is more than 6 standard deviations from zero. The normalization error is shown by the hatched band above the data points. Solid lines represent the best fits of data to obtain the values of relative amplitudes $r_{2}$ and $r_{4}$.

Figure 6 presents the $1 \sigma$ confidence level ellipses for the relative amplitudes together with their error matrix components. The real part of $r_{2}$ is well consistent with zero. At the same time, $\operatorname{Im} r_{2}$, though has a very small negative value, is different from zero by about $3.8 \sigma$.
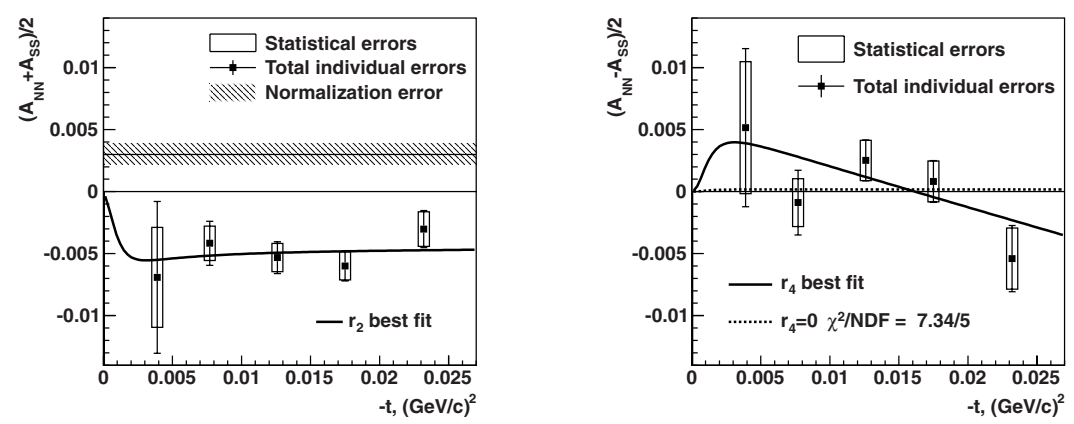

Fig. 5. Double spin asymmetries $\left(A_{N N}+A_{S S}\right) / 2$ (left) and $\left(A_{N N}-A_{S S}\right) / 2$ (right) and their fits to extract relative amplitudes $r_{2}$ and $r_{4}$. 

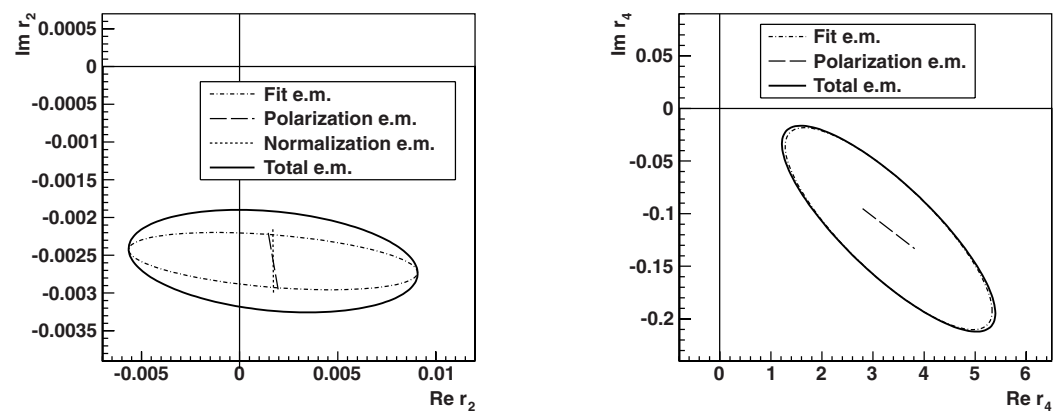

Fig. 6. Extracted values of double spin flip relative amplitudes $r_{2}$ (left) and $r_{4}$ (right) with total uncertainties and error matrix components.

Though $r_{4}$ can formally be obtained from the fit, it has large uncertainties. This is not unnatural because the corresponding helicity amplitude $\phi_{4}$ is forced to be small by the kinematic factor $t / m^{2}$ rather than by the value of $r_{4}$, see Eq. 1 . Nevertheless the $r_{4}$ deviates less than $2 \sigma$ from zero and would include zero at the confidence level of about $85 \%$. Thus the hypothesis that $r_{4} \approx 0$ cannot be excluded. To support this point, the curve with $r_{4}$ fixed to 0 was added to the right plot in Fig. 5 as a dashed line. The numerical value of $\chi^{2}=7.34$ for 5 degrees of freedom for this curve does not allow one to reject the hypothesis $r_{4} \approx 0$ as well.

\section{Conclusions}

The measured asymmetry $\left(A_{N N}-A_{S S}\right) / 2$ is compatible with zero. On the contrary the values of $\left(A_{N N}+A_{S S}\right) / 2$ are significantly below zero. Our results are at variance, both for the sign and t-dependence, with the latest predictions ${ }^{5}$ of the model based on the Regge theory. Using the extracted values of the relative double spin-flip amplitudes $r_{2}$ and $r_{4}$, we conclude that the hadronic double spin-flip amplitudes $\phi_{2}^{\text {had }}$ and $\phi_{4}^{\text {had }}$ are different at our kinematic range. This indicates that the exchange mechanism is more complex than an exchange of Regge poles only. This conclusion is further supported by comparing $\phi_{2}^{\text {had }}$ with the result on the single spin-flip amplitude $\phi_{5}^{\text {had }}$, which is found to be compatible with zero. The STAR measurements of the single and double spin asymmetries, with small uncertainties and at high energy, provide important constraints for theoretical models aiming to describe the spin-dependence of elastic scattering.

\section{References}

1. N. H. Buttimore, E. Gotsman and E. Leader, Phys.Rev. D18, 694 (1978).

2. L. Adamczyk et al., Phys.Lett. B719, 62 (2013).

3. R. Battiston et al., Nucl.Instrum.Meth. A238, p. 35 (1985).

4. C. Whitten, AIP Conf.Proc. 980, 390 (2008).

5. T. Trueman, Phys.Rev. D77, p. 054005 (2008). 